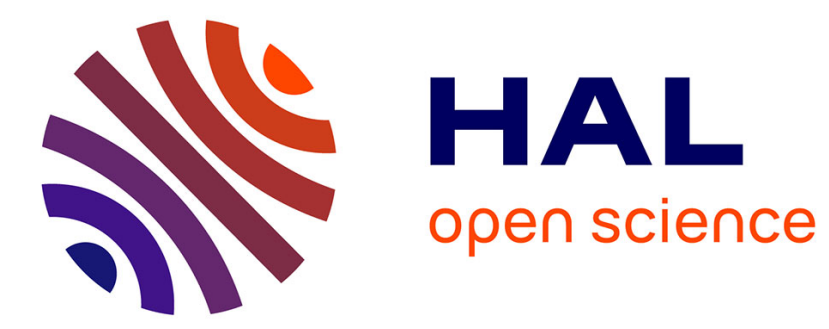

\title{
Two optic flow regulators for speed control and obstacle avoidance
}

\author{
Julien Serres, Franck Ruffier, Nicolas Franceschini
}

\section{To cite this version:}

Julien Serres, Franck Ruffier, Nicolas Franceschini. Two optic flow regulators for speed control and obstacle avoidance. The First IEEE/RAS-EMBS International Conference on Biomedical Robotics and Biomechatronics, 2006. BioRob 2006., Feb 2006, Pisa, Italy. pp.750-757, 10.1109/BIOROB.2006.1639180 . hal-02295748

\section{HAL Id: hal-02295748 \\ https://hal-amu.archives-ouvertes.fr/hal-02295748}

Submitted on 24 Sep 2019

HAL is a multi-disciplinary open access archive for the deposit and dissemination of scientific research documents, whether they are published or not. The documents may come from teaching and research institutions in France or abroad, or from public or private research centers.
L'archive ouverte pluridisciplinaire HAL, est destinée au dépôt et à la diffusion de documents scientifiques de niveau recherche, publiés ou non, émanant des établissements d'enseignement et de recherche français ou étrangers, des laboratoires publics ou privés. 


\title{
Two optic flow regulators for speed control and obstacle avoidance
}

\author{
Julien SERRES, Franck RUFFIER and Nicolas FRANCESCHINI \\ Biorobotic research group, Movement and Perception Lab., \\ CNRS/Univ. de la Méditerranée \\ 31, chemin Joseph Aiguier, 13402 Marseille Cedex 20, FRANCE \\ \{julien.serres, franck.ruffier,nicolas.franceschini\}@univmed.fr
}

\begin{abstract}
In our project on the autonomous guidance of Micro-Air Vehicles (MAVs) in confined indoor and outdoor environments, we have developed a bio-inspired optic flow based autopilot with which the speed of a miniature hovercraft is controlled and the walls of a straight or tapered corridor are safely avoided. A hovercraft is an air vehicle endowed with natural roll and pitch stabilization characteristics, in which planar flight control can be developed conveniently. Our own hovercraft is fully actuated by two rear and two lateral thrusters. It travels at a constant ground height $(\sim 2 \mathrm{~mm})$ and senses the environment by means of two lateral eyes that measure the right and left optic flows (OFs). The complete visuo-motor control system, which is called LORA(2) (Lateral Optic flow Regulation Autopilot), consists of a system of two lateral $O F$ regulators with a single $O F$ set-point:

(i) the first lateral $O F$ regulator adjusts the hovercraft's forward thrust (which determines the forward airspeed $V_{x}$ ) so as to maintain the mean value of the two (right and left) OFs measured equal to a set-point.

(ii) the second lateral $O F$ regulator controls the hovercraft's side-slip thrust (which determines the side-slip airspeed $V_{y}$ ) so as to maintain the $O F$ measured equal to the same set-point as in (i). Interestingly, this makes the distance to the left $\left(D_{L}\right)$ or right $\left(D_{R}\right)$ wall proportional to the forward airspeed $V_{x}$ determined in (i): the faster the hovercraft is travelling, the further away from the walls it will be.

Simulations have shown that the hovercraft manages to navigate in a straight or tapered corridor at speeds of up to $1 \mathrm{~m} / \mathrm{s}$ although it has only a minimalistic visual system (it is equipped with only two pixels in each eye). The passive visual sensors and the simple control system used here are suitable for use on MAVs with an avionic payload of only a few grams.

A major outcome of this work is that the LORA(2) autopilot makes the hovercraft navigate without any need for range sensors or speed sensors.
\end{abstract}

Index Terms - Optic Flow (OF), Autopilot, MAV (Micro-Air Vehicle), Hovercraft, Urban canyon navigation, Insect navigation, Biorobotics, Biomimetics, Bionics.

Abbreviations-LORA(1): the Lateral Optic flow Regulation Autopilot described in [14], LORA(2): the Lateral Optic flow Regulation Autopilot described here.

\section{INTRODUCTION}

Winged insects are able to navigate swiftly in unfamiliar environments by extracting visual information from their own motion. One of the most useful visual cues is the optic flow (OF), which is the apparent motion of the image of contrasting features projected onto the insect's retina. The OF is used by insects to avoid collisions [1,2], to follow a corridor [3], and to cruise and land [4], for example.
Based on the biorobotic approach developed at our laboratory over the past 20 years, several terrestrial and aerial vehicles equipped with $\mathrm{OF}$ sensing systems have been built [5-10], or simply simulated [11-14]. The OF sensor used for this purpose is an angular velocity sensor originally designed in $1986[15,16]$. The principle underlying this electro-optical image velocity sensor was based on findings obtained at our laboratory on the common housefly's Elementary Motion Detectors (EMDs) by performing electrophysiological recordings on single neurons while concomitantly applying optical microstimuli to two single photoreceptor cells within a single ommatidium [17].

Studies in which honeybees flying through a narrow tunnel were closely observed have shown that these insects tend to maintain a trajectory which is equidistant from the two flanking walls [3]. To explain this centring response, the latter authors proposed that the animal may balance the apparent speeds of the images of the walls perceived by their two eyes [3]. In the field of robotics, many research scientists have referred to this hypothesis when designing visually guided vehicles [18$23]$, and simulating flying agents $[24,25]$ and hovercraft $[14,26]$. The "optic flow balance" hypothesis was confirmed by performing tests in corridors and canyons. However, balancing the two lateral OFs would make these visually-guided robots rush into any opening in a wall, since openings give rise to virtually zero OF. To deal with this problem, some authors suggested switching to wall-following behaviour whenever the mean value of the two lateral OFs becomes larger than a given threshold [21] or whenever one of the two lateral OFs is equal to zero [20]. Wall-following behaviour resulted in maintaining the lateral OF constant on one side by controlling the robot's heading, which meant that at a given speed the robot would tend to maintain a "pre-specified distance" to the wall [20,21,27].

Honeybees' flight speed tends to be proportional to the local corridor width when they are travelling along a tapered corridor [4]. Some authors applied this finding to robotic systems in which the local corridor width was determined by measuring both the OF-field and the robot's groundspeed and then adjusting the groundspeed in proportion to the local corridor width $[21,26]$. Other authors used a lookup table giving the wheeled robot's groundspeed as a function of the mean OF based on the values measured on both sides [20]. Others authors again took the mean OF between the two sides in

*This research was supported by CNRS (Life Science and Engineering Science) and by a EU contract (IST/FET - 1999-29043). 
comparison with a reference value as a means of adjusting the wheeled robot's groundspeed [22].

In previous studies, we designed a bio-inspired OF based autopilot called OCTAVE, which enable a microair vehicle (MAV) to avoid the ground by making it automatically rise or descend when flying over a shallow terrain $[8,9]$. Unlike the OCTAVE autopilot, which was designed for ground avoidance, the LORA(2) autopilot (LORA stands for Lateral Optic flow Regulation Autopilot) described here affords automatic speed control in a corridor and wall collision avoidance. LORA(2) is also based on OF regulation principles and actually involves two independent OF-based airspeed control systems: (i) the first lateral OF regulator adjusts the hovercraft's forward thrust (which determines the forward airspeed $V_{x}$, i.e., the surge speed) so as to maintain the mean value of the two OFs (right and left) measured equal to a set-point - (ii) The second lateral OF regulator adjusts the hovercraft's side-slip thrust (which determines the side-slip airspeed $V_{y}$, i.e., the sway speed) so as to maintain the OF measured equal to the same set-point as in (i). The distance to the left $\left(D_{L}\right)$ or right $\left(D_{R}\right)$ wall therefore becomes proportional to the hovercraft's forward airspeed $V_{x}$ determined in (i): the faster the hovercraft travels, the further away from the walls it will be.

As a first step toward building an autopilot giving Micro-Air Vehicles (MAVs) lateral obstacle avoidance capacities, we decided to work on a miniature hovercraft because this type of air vehicle, which "flies" on a plane (at a ground height of $\sim 2 \mathrm{~mm}$ ), is endowed with inherent roll and pitch stabilization characteristics. Like flying insects and air vehicles, it makes no contact with the ground while travelling and is capable of moving forwards and sideways. The most common types of hovercraft have three degrees of freedom, X, Y, and $\Psi$. They are holonomic in the plane but usually underactuated. Our hovercraft is fully actuated, however, because it is equipped with a pair of lateral thrusters giving it side-slip motion, in addition to the pair of rear thrusters responsible for forward motion and heading control (Fig. 1). Unlike wheeled robots, and more like insects and air vehicles, hovercraft are subject to disturbances along their three degrees of freedom (such as caused by headwind, sidewind and turbulences). The hovercraft equipped with the LORA

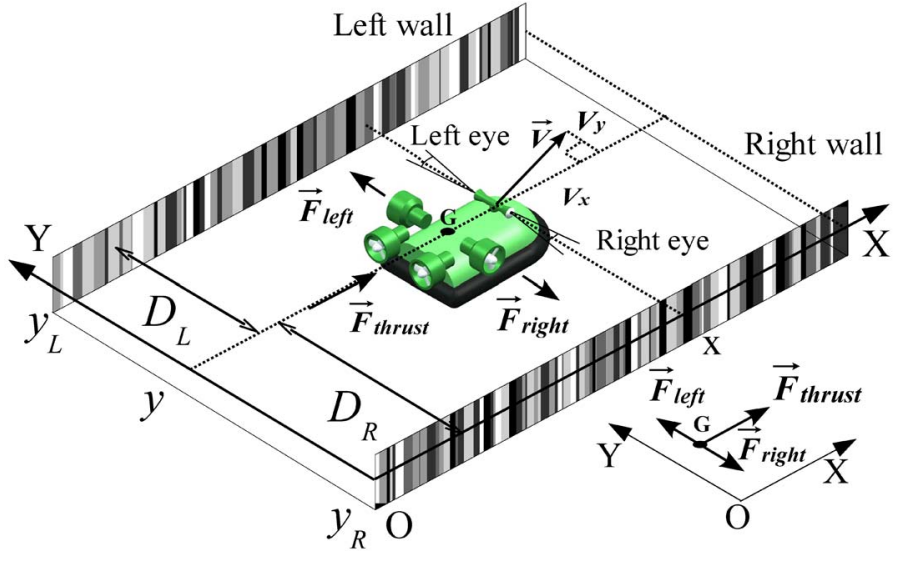

(2) autopilot is capable of performing various tasks such as wall-following and centring without having to switch abruptly from one task to another. The LORA(2) control system automatically adjusts the hovercraft's forward airspeed to the local width of the corridor, while keeping the robot at a "safe distance" from the walls. In this indoor study, since the hovercraft was not subjected to wind, its groundspeed was equal to its airspeed - but neither the groundspeed nor the airspeed is involved in the present control system anyway.

In section 2, we describe the simulation set-up used to test the two OF regulators scheme implemented on a miniature hovercraft. Section 3 focuses on the biomimetic vision-based motion control system and details of the forward-plus-lateral control loops are described. Section 4 deals with simulation runs carried out by a hovercraft equipped with the two OF regulators, which enable the robot to navigate at a relatively high speed in a straight or tapered corridor, in much the same way as honeybees are capable of doing.

\section{Simulation Set-up}

All the present experiments are computer simulations carried out on a standard PC equipped with the MATLAB $^{\mathrm{TM}} /$ SIMULINK software program.

\section{II.A. The dynamic hovercraft model}

The hovercraft travels at an airspeed $\vec{V}$ over a flat surface along a corridor with randomly textured walls in terms of both the spatial frequency and the contrast (Fig. 1). In the 2-D approximations used throughout this paper, the hovercraft's motion is defined by dynamic equations involving the forward thrust $\left(F_{\text {thrust }}\right)$ produced by the rear thrusters and the side-slip thrusts $\left(F_{\text {left }}\right.$ and $\left.F_{\text {right }}\right)$ produced by the lateral thrusters (see Fig. 1, inset). The propeller dynamics is neglected here. The following linearized system of equations referred to the center of gravity $\mathrm{G}$ is used:

$$
\begin{gathered}
m . d V_{x} / d t+\zeta_{x} . V_{x}=F_{\text {thrust }} \\
m . d V_{y} / d t+\zeta_{y} \cdot V_{y}=\text { Fleft }-F_{\text {right }}
\end{gathered}
$$

where $m$ is the mass of the hovercraft, and $\zeta_{x}$ and $\zeta_{y}$ are translational viscous friction coefficients along the $\mathrm{X}$ axis and the Y-axis, respectively. The hovercraft is both holonomic and fully actuated: each of the airspeed components $V_{x}$ and $V_{y}$ (Eq. 1 and Eq. 2) is controlled independently. In this study, the hovercraft's heading is assumed to be stabilized along the $\mathrm{X}$-axis by implementing a heading lock system based, for example, on a micro gyro-compass. This heading lock system is intended to compensate for any yaw disturbances by controlling the two rear thrusters differentially.

Figure 1. Miniature hovercraft travelling at airspeed $\vec{V}$ along an unfamiliar textured corridor. The figure in the inset shows the forces acting on the vehicle and shows their application points. Four thrusters on the hovercraft control the three degrees of freedom in the plane independently. The forward airspeed $V_{x}$ results from the total thrust $\left(F_{\text {thrust }}\right)$ exerted on the two rear thrusters (Eq. 1). The side-slip airspeed $V_{y}$ results from the combined force of the two lateral thrusters $\left(F_{l e f t}\right.$ and $\left.F_{\text {right }}\right)$, as described by Eq. 2 . The vehicle's heading is maintained along the X-axis by means of a heading lock system (e.g., based on a gyro-compass servo) that compensates for any yaw disturbances by adjusting the two rear thrusters differentially. 
II.B. Optic flow (OF) generated by the hovercraft's own motion

The OF is the apparent angular velocity of the environmental features projected onto the two (diametrically opposed) lateral eyes. The eyes receive a right and a left OF, $\omega_{R}$ and $\omega_{L}$, respectively, which can be defined as follows (Fig. 1):

$$
\begin{aligned}
& \omega_{R}=V_{x} / D_{R} \\
& \omega_{L}=V_{x} / D_{L}
\end{aligned}
$$

where $V_{x}$ is the hovercraft's forward airspeed, and $D_{R}$ and $D_{L}$ are the distances from the right and left walls, respectively. Since any rotation is compensated for (see section II.A), each eye receives a purely translational OF.

\section{II.C. Simulation of the optic flow sensors}

Two lateral eyes are mounted in diametrically opposed positions at a right angle with respect to the hovercraft's symmetry axis (Fig. 1). Each eye consists of only two photoreceptors (two pixels), the visual axes of which are separated by an interreceptor angle $\Delta \varphi=4^{\circ}$. The angular sensitivity of each photoreceptor is a bell-shaped function where the acceptance angle (the angular width at half height) is also $\Delta \rho=4^{\circ}[28]$. The principle underlying the Elementary Motion Detector (EMD) serving as an OF sensor has been previously described $[7,15,16,28]$. It is a nonlinear electronic circuit driven by the two photoreceptors, and its output is a monotonic function (within a 10 -fold range from $40 \%$ s to $400 \%$ s) of the angular velocity of a moving texture [9]. The output signal of each photoreceptor is computed at each time step by summing together all the grey level patterns present in its field of view (which covers approximately three $\Delta \rho$, i.e., $12^{\circ}$ ) and weighing the result of the summation with a bell-shaped angular sensitivity function.

\section{The LORA(2) Autopilot}

The hovercraft is controlled by an autopilot called LORA(2) (Lateral Optic flow Regulation Autopilot). The latter involves multiple processing steps, as shown in Fig. 2. Two independent visuomotor feedback loops have been implemented to control the hovercraft's motion along the two translational degrees of freedom $X$ and $\mathrm{Y}$. The hovercraft reacts to variations in the lateral OF by selectively adjusting the two orthogonal components $V_{x}$ and $V_{y}$ of its airspeed. A forward visuomotor feedback loop interprets any increase in the lateral $\mathrm{OF}$ as an acceleration, and a side-slip visuomotor feedback loop interprets any increase in the lateral OF as a decrease in the distance from the left or right wall.

\section{III.A. Forward speed visuomotor feedback loop}

\section{III.2) Bio-inspiration}

This guidance strategy was inspired by findings obtained on the flight behaviour of honeybees in a small tapered corridor lined with periodic black-and-white vertical stripes [4]. The authors of the study in question observed that for a bee centred upon entering the corridor, the flight speed tends to be proportional to the local width of the corridor: the insects slowed down as they approached the narrowest section and accelerated when the corridor widened beyond this point. The authors concluded that "the speed of the flight is controlled by regulating the image velocity" [4].

\section{III.3) Forward speed dynamics}

The dynamic model $\boldsymbol{G}_{\boldsymbol{V} x}(\mathbf{s})$ for our hovercraft (from Eq. 1), which relates the forward airspeed $V_{x}$ to the forward control signal $U_{\text {forward, }}$ (Fig. 2), is described as a first order low-pass filter with a time constant of $0.5 \mathrm{~s}$ (Eq. 5):

$$
G_{V_{x}}(s)=\frac{V_{x}(s)}{U_{\text {forward }}(s)}=\frac{0.1}{1+0.5 s}
$$

\section{III.A.1) Forward speed visual feedback loop}

The forward speed autopilot is the first $O F$ regulator. The feedback signal it receives is the mean value of the right $\left(\omega_{\text {Rmeas }}\right)$ and left ( $\left.\omega_{\text {Lmeas }}\right)$ OFs measured (Fig. 2 , blue upper loop). The autopilot was designed to keep the mean value of the two lateral OFs measured constant by adjusting the forward thrust (which determines the forward airspeed). Any increase in the mean value of the two lateral OFs is interpreted as resulting from the hovercaft's acceleration. This control scheme thus automatically ensures a "safe forward speed". The error $\varepsilon_{\text {forward }}$ in the input to the forward controller (see Fig. 2) is computed as follows:

$$
\varepsilon_{\text {forward }}=\omega_{\text {SET }}-\left(\omega_{R_{\text {meas }}}+\omega_{\text {Lmeas }}\right) / 2
$$

The mean value of the two OFs measured is compared with an OF set-point $\omega_{S E T}$ (Eq. 6). A proportional forward controller with a gain $U_{\text {forward }} / \varepsilon_{\text {forward }}$ of 50 was introduced into the feedback loop to improve the closedloop dynamics.

\section{III.B. Side-slip visuomotor feedback loop}

\section{III.B.1) Bio-inspiration}

The side-slip control principle described was inspired by findings made on the flight behaviour of honeybees [3]. The authors of the latter study observed that honeybees tend to fly along the midline of a straight corridor (centring response), and concluded that "bees maintained equidistance by balancing the velocities of the retinal images in the two eyes" [3].

III.B.2) Side-slip dynamics

As derived from Eq. 2, the transfer function $G_{y}(\mathbf{s})$ relating the hovercraft's ordinate $y$ to the control signal 
$U_{\text {side, }}$ approximates a first-order low-pass filter (with a time constant of $0.5 \mathrm{~s}$ ) in series with an integrator (Eq.7).

$$
G_{Y}(s)=\frac{\mathrm{Y}(\mathrm{s})}{\mathrm{U}_{\text {side }}(s)}=\frac{1}{s}\left(\frac{0.1}{1+0.5 s}\right)
$$

\section{III.B.3) Side-slip visual feedback loop}

The side-slip autopilot is the second $O F$ regulator. The feedback signal it receives is the largest of the two measured OFs (left and right OFs). This autopilot (Fig. 2 , red bottom loop) was designed to keep the lateral OF constant and equal to an OF set-point $\omega_{S E T}$ which is identical to that used in the forward speed autopilot. The hovercraft then reacts to any changes in the lateral OF by adjusting the side-slip thrust (which determines the hovercraft's side-slip airspeed $V_{y}$ ), thus adjusting the distance from the left $\left(D_{L}\right)$ or right $\left(D_{R}\right)$ wall. A sign function automatically selects the wall that will be followed. For this purpose, a maximum criterion is used to select the higher OF value measured between the right OF $\left(\omega_{\text {Rmeas }}\right)$ and the left OF $\left(\omega_{\text {Lmeas }}\right)$, which provides information about the nearest wall. The selected OF value is compared with the OF set-point $\omega_{S E T}$ (Fig. 2, red bottom loop). In the steady state, the selected OF will therefore become equal to the set-point $\omega_{S E T}$. The error $\varepsilon_{\text {side }}$ in the input to the side-slip controller is computed as follows:

$$
\varepsilon_{\text {side }}=\operatorname{sign}\left(\omega_{\text {Lmeas }}-\omega_{R_{\text {meas }}}\right) \times\left(\omega_{\text {SET }}-\max \left(\omega_{R_{\text {meas }}}, \omega_{\text {Lmeas }}\right)\right)
$$

A lead controller $\boldsymbol{C} \boldsymbol{v}_{y}(\mathbf{s})$ (Eq. 9) was introduced into this feedback loop to increase the damping, thus improving the stability and response dynamics.

$$
C_{V y}(s)=\frac{U_{\text {side }}(s)}{\varepsilon_{\text {side }}(s)}=\frac{1+2.5 s}{1+0.2 s}
$$

\section{Simulation Results}

\section{IV.A. Straight corridor following}

\section{IV.A.1) Simulated visual environment}

The simulated visual environment is a 4-meter long, 1meter wide straight corridor. Its right and left walls are lined with a random pattern of various grey vertical stripes covering a large spatial frequency range (from $0.069 \mathrm{c} /{ }^{\circ}$ to $0.87 \mathrm{c} /{ }^{\circ}$ reading from the midline) and a large contrast range (from $6 \%$ to $40 \%$ ). No special steps were taken to make the two opposite patterns mirrorsymmetric.

\section{IV.A.2) Side-slip control only}

First we examine the case of a single (side-slip) control of the hovercraft, where the forward airspeed $V_{x}$ is set at $1 \mathrm{~m} / \mathrm{s}$. It can be seen from Fig. 3a (bold curve) that the resulting trajectory is highly reminiscent of the bee's "centring response". For the sake of comparison, the dotted curve in Fig. 3a gives the trajectory obtained with a previous autopilot, which we called LORA(1) [14], where the hovercraft's yaw velocity $(\mathrm{d} \Psi / \mathrm{d} t)$ was controlled on an OF basis (while the airspeed $V=\sqrt{V_{x}^{2}+V_{y}^{2}}$ was kept at $1 \mathrm{~m} / \mathrm{s}$ ). The dotted trajectory clearly shows the oscillations which occurred due to the fact that the lateral OFs measured were proportional only to the Xcomponent $V_{x}$ of the airspeed. Hence, the forward airspeed $V_{x}$ was not constant and also oscillated (see Fig. $3 b)$.

\section{IV.A.3) Side-slip-plus-forward control}

Figure 4a shows the robot trajectories resulting from the LORA(2) control scheme based on two OF regulators, each one controlling one degree of freedom, X or Y. It can be seen from Fig. 4a that whatever its initial position at the entrance to the straight corridor, the hovercraft navigates safely. The two OF regulators immediately

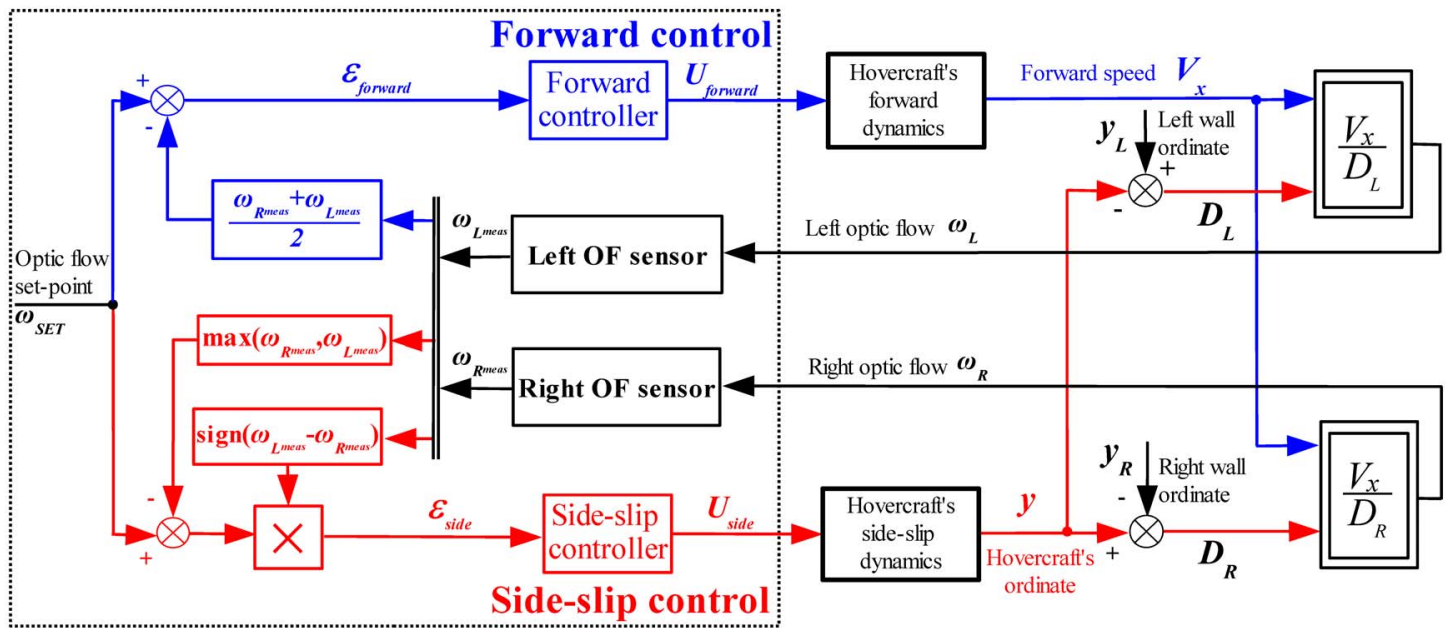

\section{LORA(2) AUTOPILOT}

Figure 2. The LORA(2) autopilot is based on two OF regulators with a single OF set-point $\omega_{\text {SET. }}$ LORA(2) regulates the lateral OF via two visual feedback loops: the forward airspeed control system (upper blue loop) and the side-slip airspeed control system (bottom red loop). The forward controller adjusts the forward thrust (which determines the hovercraft's airspeed $\left.V_{x}\right)$ on the basis of the mean value of the right and left OFs measured: $\left(\omega_{\text {Rmeas }}+\omega_{\text {Lmeas }}\right) / 2$. This mean value is compared with an OF set-point $\omega_{S E T}$. The forward controller commands the forward motion so as to minimize $\varepsilon_{\text {forward }}$. The side-slip controller adjusts the side-slip thrust (which determines the hovercraft's ordinate $y$ ) on the basis of whichever of the two lateral OFs is the largest. The latter value is compared with the same OF set-point $\omega_{S E T}$, and the direction of avoidance is given by the sign of the difference between the left and right OFs measured. The side-slip controller commands the side-slip motion so as to minimize the error $\varepsilon_{\text {side }}$. 
react to the feedback information about the nearest wall. For a corridor width of $1 \mathrm{~m}$, the autopilot brings the craft back close to the midline.

\section{IV.A.4) Effect of the forward visual feedback loop}

The beneficial effects on the response kinetics of providing each airspeed component $V_{x}$ and $V_{y}$ with an independent visual control can be seen from Fig. 4. LORA(2) is more reactive than the previous version [14] because the forward airspeed is now also controlled on the basis of the lateral OF. But the higher the forward airspeed $V_{x}$, the larger the overall feedforward gain of the second side-slip control loop will be (see Fig. 2, bottom red loop), because $\omega_{R}$ and $\omega_{L}$ are proportional to the forward airspeed $V_{x}$ (Eq. 3,4). Hence, the faster the hovercraft travels, the more reactive the side-slip visuomotor feedback loop will be.

\section{IV.A.5) Effect of a "no contrast" zone}

Figure 5 shows the effect of a local absence of contrast on one wall. This "no contrast" zone simulates a gap in the texture or an opening. Although it is unable to measure any OF along the 1.5-meter long aperture on its left hand side, the hovercraft automatically follows the opposite (right) wall. At first, from $X=1.6 \mathrm{~m}$ to $X=2.2 \mathrm{~m}$, the hovercraft is not affected by the "no contrast" zone because the left OF sensor holds the last measured OF for another $0.5 \mathrm{~s}$. Once this hold time has elapsed, the hovercraft follows the right wall, due to the automatic change in the sign of the error $\varepsilon_{\text {side }}$ (Fig. 2 and Eq. 8), which makes the craft respond to the right wall only. Acceleration occurs transiently because the environment is less cluttered, which reduces the mean value $\left(\left(\omega_{\text {Rmeas }}+\omega_{\text {Lmeas }}\right) / 2\right)$ of the two lateral OFs measured (see Fig. 2).

\section{IV.B. Tapered corridor following}

The simulated visual environment used here (Fig. 6) is a 12-meter long tapered corridor with a 2-meter wide entrance and a 0.5 -meter wide constriction located midway. The patterns on the walls are the same as in the previous experiments.

\section{IV.B.1) Automatic deceleration and acceleration}

It can be seen from Fig. 6a that the hovercraft automatically slows down as it approaches the narrowest section of the corridor and accelerates when the corridor widens beyond this point. The robot therefore negotiates a narrow passage by automatically decelerating (and it accelerates once it has passed the constriction). Figure $6 \mathrm{a}$ also shows that centring behaviour is not systematic but depends on the initial Y-position at the entrance to the corridor. Figure $6 \mathrm{~b}$ shows that once the hovercraft has passed the constriction point, it is quasi centred, and hence its forward airspeed is automatically ajusted to the local corridor width.

\section{IV.B.2) Effect of saturation signals}

The lateral control signal $U_{\text {side }}$ was bounded so as to limit the maximum side-slip airspeed to $\pm 0.2 \mathrm{~m} / \mathrm{s}$, and the
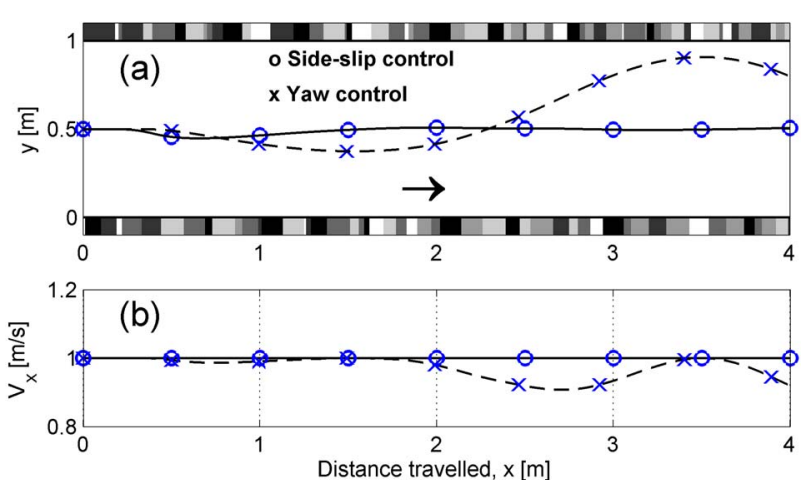

Figure 3. Side-slip control versus yaw control (Marks on the trajectories indicate the hovercraft position at $0.5 \mathrm{~s}$ intervals). (a) Two simulated trajectories of the hovercraft moving to the right (arrow) at a constant speed $(V=1 \mathrm{~m} / \mathrm{s})$ in a onemeter wide straight corridor at an OF set-point $\omega_{\text {SET }}$ equal to $80 \%$ with two different control laws. Solid curve: the robot is piloted by a side-slip control system $\left(V_{y}\right)$, whereby $V_{x}$ is kept at $1 \mathrm{~m} / \mathrm{s}$. Dotted curve: the hovercraft is piloted by a yaw control system (see LORA(1) in [14]). (b) The forward airspeed $V_{x}$ of the hovercraft controlled by the yaw system (crosses) can be seen to vary, thus affecting the measurement of the lateral OF (see Eq. 3 and Eq. 4). Oscillations appear on the curve. By contrast, a side-slip control system of the hovercraft maintains the forward speed constant (open dots) and the oscillations disappear.
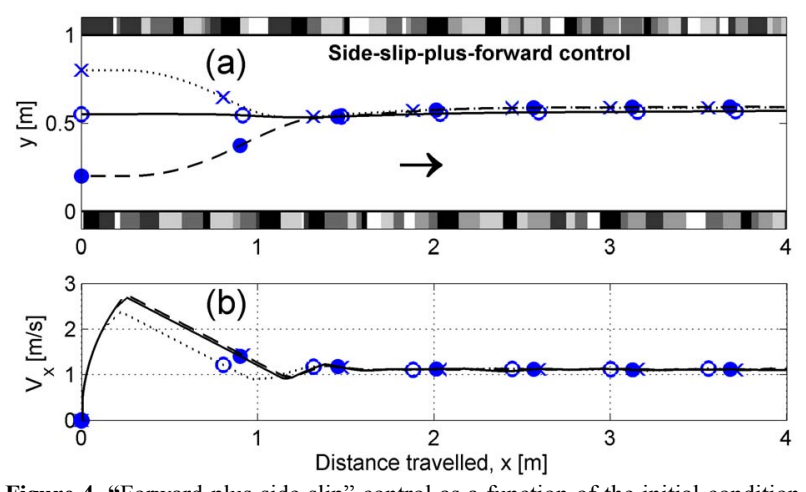

Figure 4. "Forward-plus-side-slip" control as a function of the initial condition (Marks on trajectories indicate the hovercraft position at $0.5 \mathrm{~s}$ intervals). (a) Three simulated trajectories of the hovercraft moving to the right (arrow) in a straight corridor at an OF set-point $\omega_{S E T}$ equal to $150^{\circ} \mathrm{s}$, from different initial positions (crosses: $\mathrm{Y}=0.8 \mathrm{~m}$, open dots: $\mathrm{Y}=0.55 \mathrm{~m}$, full dots: $\mathrm{Y}=0.2 \mathrm{~m}$ ). The hovercraft can be seen to navigate safely whatever the initial position. (b) Forward speed profiles corresponding to the three trajectories shown in (a). In the steady state, the forward airspeed reached in the three simulated cases is $1 \mathrm{~m} / \mathrm{s}$.
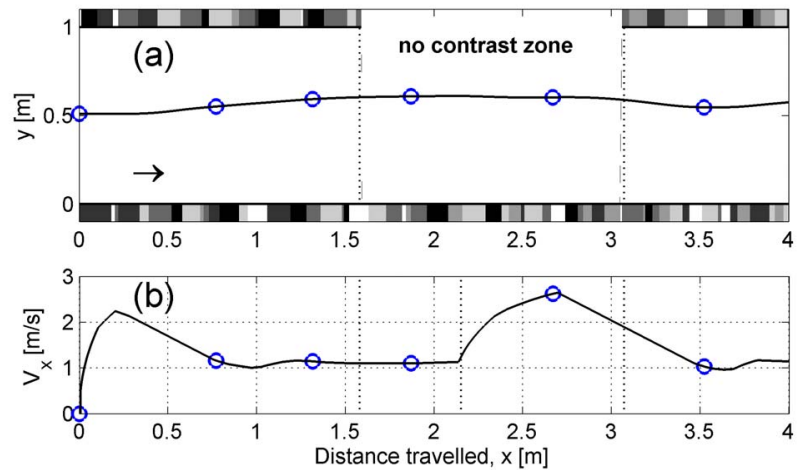

Figure 5. Effect of a local absence of texture on one wall. $\omega_{S E T}=150 \%$ (Marks on trajectories indicate the hovercraft position at $0.5 \mathrm{~s}$ intervals). (a) Simulated trajectory showing that the robot is hardly affected by a 1.5 -meter long "no contrast" zone along the left wall. The LORA(2) autopilot makes the hovercraft automatically catch on to the right wall and follow it, because $\varepsilon_{\text {forvard }}$ (Eq. 6) changes sign automatically as the left OF becomes equal to zero (see Fig. 2). (b) Forward speed profile along the corridor. At the start, we observe the step response of the two OF regulators: the forward airspeed increases transiently before reaching a steady state at about $1 \mathrm{~m} / \mathrm{s}$. As the motion is disturbed by the "no contrast" zone, the forward speed increases until the mean value of the two OF measured reaches the OF set-point. The delay observed in the reaction to the "no contrast" zone is due to the last OF measurement on the left hand side persisting for $0.5 \mathrm{~s}$. 

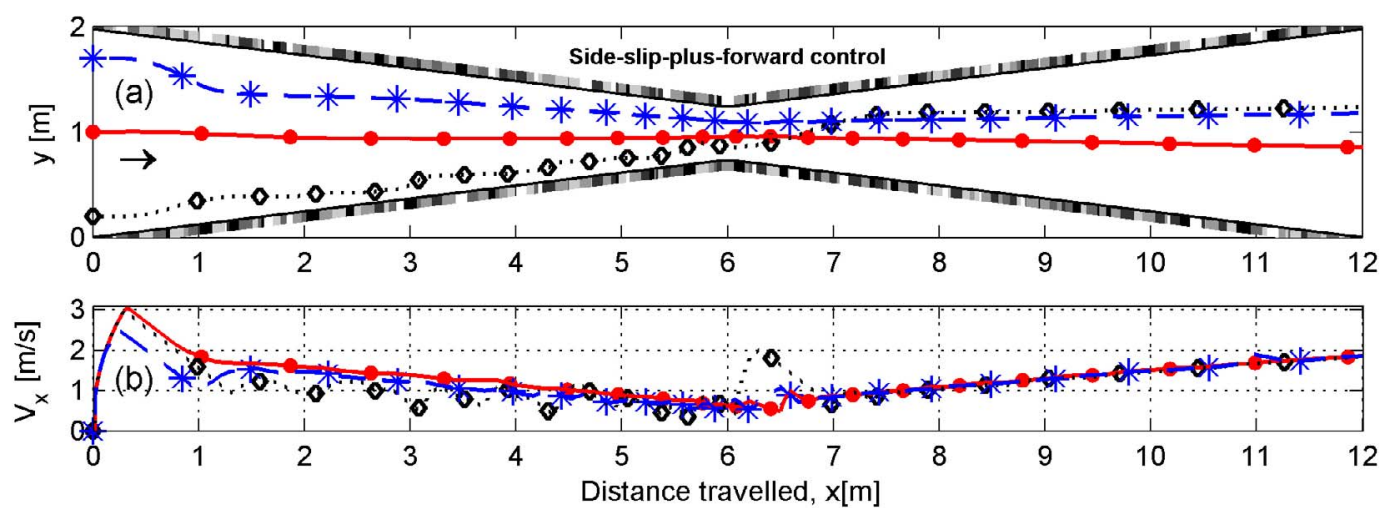

Figure 6. "Side-slip-plus-forward" control with three different initial positions (Marks on trajectories indicate the hovercraft position at $0.5 \mathrm{~s}$ intervals). (a) Three simulated trajectories of the hovercraft moving to the right (arrow) in a tapered corridor. The OF set-point value $\omega_{S E T}$ is $150^{\circ}$ s. These trajectories show that the hovercraft slows down when the corridor width decreases and accelerates when it widens. (b) The forward speed control system makes the hovercraft automatically generate a safe forward speed. Once it has passed the constriction point, the hovercraft is quasi-centred. From then on, The hovercraft's forward airspeed is therefore a linear function of the distance travelled $\mathrm{x}$, it is also proportional to the corridor width. When the hovercraft approaches a wall, its distance from the wall is proportional to its forward speed.
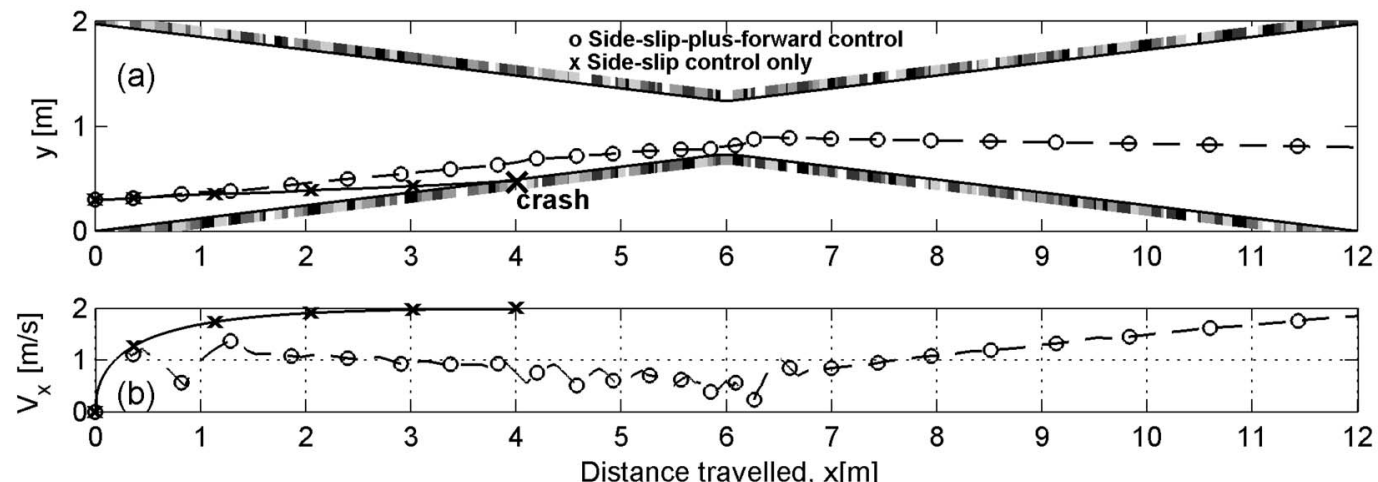

Figure 7. "Side-slip control" versus "side-slip-plus-forward control" with a more realistic model including saturated control signals that impose limitations on the actuators (Marks on trajectories indicate the hovercraft position at $0.5 \mathrm{~s}$ intervals). (a) In a "side-slip control only" case, the robot eventually collides with the right wall; whereas with two OF regulators that control both the forward and side-slip speeds, the lateral contol signal $U_{\text {side }}$ does not saturate and the robot negociates the tapered corridor safely (open dots). (b) The hovercraft's forward airspeed as a function of the distance travelled. The forward speed is limited to $2 \mathrm{~m} / \mathrm{s}$.

control signal $U_{\text {forward }}$ was also bounded so as to limit the forward airspeed to $2 \mathrm{~m} / \mathrm{s}$. Under these conditions, the forward speed control system has beneficial effects as shown in Fig. 7. Without any visual feedback about the forward airspeed (crosses), the hovercraft soon collides with the right wall (Fig. 7: cross marks); once equipped with the complete LORA(2) control system the hovercraft negotiates the tapering passage successfully, starting from various initial positions.

IV.B.3) Forward speed under lateral optic flow regulation when the craft is initially centred

Figure 8a again shows the quasi centred trajectory of Fig. 6a to illustrate the effect of the LORA(2) autopilot on the hovercraft's behaviour. Figure $8 \mathrm{~b}$ shows that when the craft is initially centred, the forward airspeed $V_{x}$ is a linear function of the distance $x$ along the whole tapered corridor, and that it is therefore also proportional to the local corridor width. This result was obtained thanks to LORA(2) autopilot, i.e., thanks to the two $O F$ regulators scheme. In the appendix, it is established that the forward airspeed inevitably decreases (and then increases) exponentially with time, with a time constant that depends on the OF value $\omega_{0}$ actually maintained and on the tapering angle $\alpha$ of the corridor $\left(\alpha=7^{\circ}\right.$ in Fig. $8 \mathrm{a})$. Plotting the forward airspeed $V_{x}$ as a function of the distance travelled $x$ defines a phase plane (Fig. 8b) in which the time constant of the exponential decay can be read directly. This time constant is equal to $1 /\left(\omega_{0} \times \tan (\mid)\right.$ $\alpha \mid)$ ) where $\omega_{0}$ is the OF that is maintained constant throughout the tapered corridor. The results of simulations show that the lateral OF was held here at $\omega_{0}=120 \%$ s. A good match can be observed between the OF $\left(\omega_{0}=120 \%\right.$ s $)$ that is actually maintained by the hovercraft and the OF $\left(\omega_{0}=106 \%\right.$ s $)$ computed from Eq. 13 (see Appendix) with the parameter identified by the linear regression curve (blue line through the data points in Fig. 8b).

The tapered corridor is taken by the LORA(2) autopilot (Fig. 2) to be a "ramp disturbance" (linear changes of left and right wall ordinates, $y_{L}$ and $y_{R}$, respectively), which makes the hovercraft respond with a linearly decreasing forward speed. The error in the ramp following performances is due here to the forward speed controller being a simple gain.

\section{Discussion and Conclusion}

The results of the present simulations show how the combined effects of two lateral OF regulators controlling the translational degrees of freedom $\mathrm{X}$ and $\mathrm{Y}$ independently can be used to enable a hovercraft to navigate in a straight or a tapered corridor under visual control. 

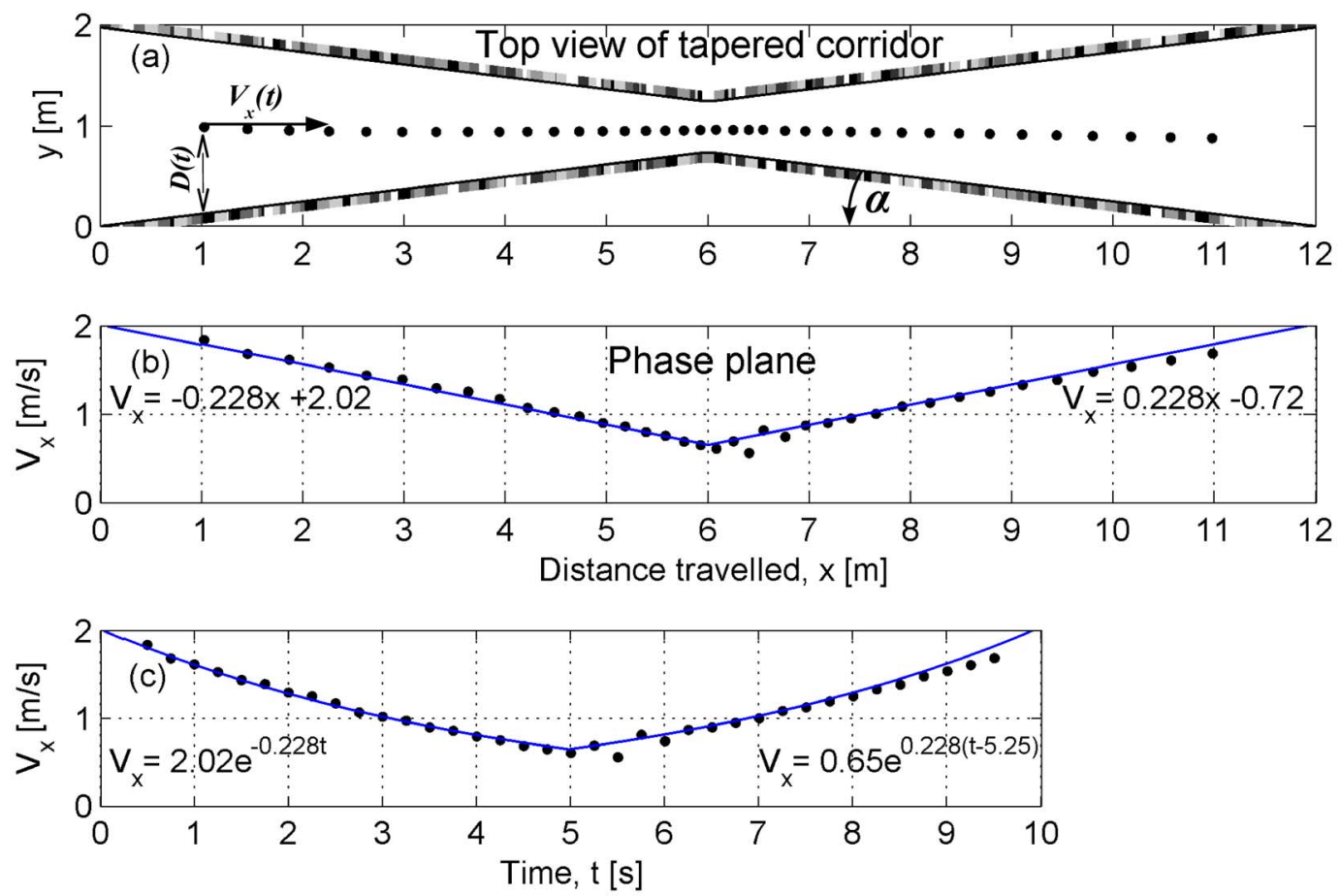

Figure 8. Forward speed profile in a tapered corridor for the quasi centred trajectory shown in Fig. 6a (Marks on trajectories indicate the hovercraft position at $0.25 \mathrm{~s}$ intervals). (a) Simulated trajectory of the hovercraft moving to the right in a tapered corridor at an OF set-point value $\omega_{S E T}$ of $150^{\circ} / \mathrm{s}$. The two $O F$ regulators (Fig. 2) make the hovercraft automatically slow down when the corridor width decreases and automatically accelerate again when the width increases. (b) Phase plane plot of the forward airspeed $V_{x}$ as a function of the distance $x$ along the corridor. The forward airspeed $V_{x}$ turns out to be a linear function of the distance $x$. The slope of the linear regression curve (blue line) is equal to the inverse of the time constant (the latter is $4.4 \mathrm{~s}$ here). (c) The forward speed is an exponential function of time, as was to be expected from the phase plane in (b).

The vision-based LORA(2) autopilot described here is based on two OF regulators with the same OF setpoint: (i) the first lateral $O F$ regulator adjusts the forward thrust (which determines the hovercraft's forward speed $V_{x}$, i.e., the surge speed) so as to keep the mean value of the two OFs measured (right and left) equal to a set-point - (ii) the second lateral $O F$ regulator adjusts the hovercraft's side-slip thrust (which determines the side-slip speed $V_{y}$, i.e., the sway speed) so as to maintain the OF measured equal to the same setpoint as in (i). Interestingly, the distance to the left $\left(D_{L}\right)$ or right $\left(D_{R}\right)$ wall becomes proportional to the hovercraft's forward speed $V_{x}$ determined in (i): the faster the hovercraft is travelling, the further away from the left (or right) wall it will be. The combined visuomotor loops thus automatically ensure a "safe groundspeed" and a "safe distance".

Like honeybees in flight, the hovercraft makes no contact with the ground while travelling and can be disturbed by wind. In spite of the minimalistic number of OF sensors with which it is equipped (one on the right and one on the left), our control scheme accounts remarkably well for the behaviour observed in honeybees flying centred along a tapered corridor, where they were found to hold the angular velocity of the image of the surroundings reaching the lateral eye region constant (Fig. 2 in [4]). From figure 2C [4], we can graphically assess the value of the lateral OF that was maintained by the honeybees in the tapered corridor (tapering angle $\alpha=15^{\circ}$; slope of the regression line equal to 1.5 based on figure $2 \mathrm{C}$ [4]). This value was about $320 \%$, which fits the value predicted on the basis of
Eq. 13 (see Appendix) and the data in [4, Fig. 2C] and the mean left and right OF $\left(317^{\circ} / \mathrm{s}\right.$ and $388 \%$, respectively) that were actually experienced by the honeybees inside the tapered corridor (as computed from the data in figure 2B [4]). The control scheme described here (Fig. 2) therefore generates data that are uncannily similar to those measured in real insects, which suggests that a similar control scheme may well be implemented in the insect nervous system.

The LORA(2) autopilot can be applied to other types of air vehicles such as blimps and helicopters. This OF-based autopilot needs to be improved by enhancing the visual viewfield and devising a means of controlling the third degree of freedom (yaw) visually to enable the robot to negotiate more challenging corridors including L-junctions or $\mathrm{T}$-junctions successfully. The additional vision-based control module required for this purpose could be achieved by comparing the two lateral parts of the OF-field, as flies seem to do when they trigger body saccades $[1,2,27]$. Studies on the implementation of the overall LORA autopilot on a fully actuated miniature hovercraft are now under way.

Insect inspired visual systems can yield solutions requiring a much smaller number of pixels than those used in the present-day computer-vision systems harnessed to mobile robots. We have described here how a robot can navigate safely in unfamiliar, and even challenging environments such as a tapered corridor, using a minimalistic number of pixels (only four pixels in fact) without any metric sensors such as rangefinders or velocimeters. Combined with an $O F$ regulator operating in the vertical plane to ensure ground obstacle 
avoidance [8,9], the two $O F$ regulators scheme presented here is one step towards low-cost visual guidance systems for autonomous vehicle navigation in unfamiliar indoor and outdoor environments. Passive OF sensors with a simple processing system will be particularly suitable for use with Micro-Air Vehicles (MAVs), which impose highly stringent constraints in terms of the avionic payload and the onboard energy resources allowed.

\section{APPENDIX}

Here, we focus on the forward speed profile along the tapered corridor (Fig. 8b). The translational lateral OF is assumed to be perfectly regulated along the whole tapered corridor. This lateral $\mathrm{OF}$ is maintained at a constant level $\omega_{0}$ :

$$
\frac{V x(t)}{D(t)}=\omega_{0}
$$

The geometric relation linking the lateral and forward motions (see Fig. 8a) is therefore $D(t+d t)-\mathrm{D}(t)=\tan (\alpha) \cdot V_{x}(t) \cdot d t$. By differentiating Eq. 10, the forward speed is the solution of the following first-order differential equation:

$$
\frac{d V_{x}(t)}{d t}-\tan (\alpha) . \omega_{0 .} V_{x}(t)=0
$$

and hence, $V_{x}$ is an exponential function of time:

$$
V_{x}(t)=V_{x}\left(t_{0}\right) \cdot e^{\tan (\alpha) \cdot \omega_{0} \cdot(t-t 0)}
$$

This means that the hovercraft is bound to slow down as it enters a narrowing section $(\alpha<0)$, its forward speed will decrease as an exponential function of time (Eq. 12, time constant $1 /\left(\tan |\alpha| \times \omega_{0}\right)$ ), and incease again exponentially (with the same time constant) when the sections widens $(\alpha>0)$. The distance travelled $x$, which is the integral over time, therefore will also decrease and increase as an exponential function of time. Since both speed $\mathrm{d} x / \mathrm{dt}$ and distance $x$ decline and grow with the same function of time, a plot of $\mathrm{d} x / \mathrm{dt}$ versus $x$ must give a linear function (Eq. 13) in the phase plane $(x, \mathrm{~d} x / \mathrm{d} t)$, as was actually observed experimentally (Fig. 8b).

$$
V_{x}(x)=\tan (\alpha) \cdot \omega_{0} \cdot x+V_{0}
$$

In a straight corridor $\alpha=0$, the hovercraft's forward speed will remain constant, as was also observed experimentally (Fig. $4 b)$.

\section{ACKNOWLEDGEMENTS}

We are grateful to S. Viollet for his fruitful comments and suggestions during this study, to M. Boyron and F. Paganucci for their expert technical assistance, to J. Blanc for her help with the English manuscript and to the three anonymous Referees for their valuable comments.

\section{REFERENCES}

[1] H. Wagner, "Flow-field variables trigger landing in flies". Nature, no. 297, pp. $147-148,1982$

[2] L.F. Tammero, and M.H. Dickinson, "The influence of visual landscape on the free flight behavior of the fruit fly drosophila melanogaster". J. Exp. Biol., no. 205, pp. 327-343, 2002.

[3] W.H. Kirchner, and M.V. Srinivasan, "Freely flying honeybees use image motion to estimate object distance". Naturwissenschaften, no. 76, pp. 281-282, 1989.M.V.

[4] Srinivasan, S.W. Zhang, M. Lehrer, and T.S. Collett, "Honeybee navigation en route to the goal: visual flight control and odometry". J. Exp. Biol., no. 199, pp. 237-244, 1996.

[5] N. Franceschini, J.M. Pichon, and C. Blanes, "From insect vision to robot vision”. Phil. Trans. R. Soc. Lond. B, no. 337, pp. 283-294, 1992.
[6] F. Mura, and N. Franceschini, "Obstacle avoidance in a terrestrial mobile robot provided with a scanning retina". Intelligent Vehicles II. N.Aoki and I. Masaki, Eds., M.I.T. Press, Cambrige, U.S.A., pp. 47-52, 1996.

[7] S. Viollet, and N. Franceschini, "Visual servo system based on a biologically-inspired scanning sensor". In Proceedings of SPIE Conf. on Sensor Fusion and Decentralized Control on Robotics II, Boston, U.S.A., vol. 3839, pp. 144-155, 1999.

[8] F. Ruffier, and N. Franceschini, "OCTAVE, a bioinspired visuo-motor control system for the guidance of micro-air vehicle". In Proceedings of SPIE conf. on Bioengineered and Bioinspired Systems (Maspalomas, Spain, May 19-21). Eds Rodriguez-Vazquez, Abbott, Carmona, Bellingham, U.S.A., vol. 5119, pp. 1-12, 2003.

[9] F. Ruffier, and N. Franceschini, "Optic flow regulation: the key to aircraft automatic guidance". Robotics and Autonomous Systems, vol. 50, Issue 4, pp. 177-194, 2005.

[10] S. Viollet, and N. Franceschini, "A high speed gaze control system based on the vestibulo-ocular Reflex". Robotics and Autonomous Systems, vol. 50, Issue 4, pp. 147-161, 2005

[11] F. Mura, and N. Franceschini, "Visual control of altitude and speed in a flying agent". In From Animals to animats III, D. Cliff et al., Eds., MIT Press, Cambridge, U.S.A., pp. 91-99, 1994.

[12] Martin N. and N Franceschini, "Obstacle avoidance and speed control in a mobile vehicle equipped with a compound eye". In: Intelligent Vehicles MIT Press, Eds L. Masaki, Cambridge, pp. 381-386, 1994.

[13] F. Ruffier, "Pilote automatique biomimétique. Système générique inspiré du contrôle visuomoteur des insectes pour: le suivi de terrain, la réaction au vent et l'atterrissage automatiques d'un micro-aéronef'. $\mathrm{PhD}$ thesis (in French), Institut National Polytechnique, Grenoble, 2004.

[14] J. Serres, F. Ruffier, and N. Franceschini, "Biomimetic visual navigation in a corridor: to centre or not to centre?". In Proceeedings of Int. Mediterranean Modeling Multiconference (Marseilles, France, Oct. 2022), pp. 91-97, 2005.

[15] C. Blanes, "Appareil visuel élémentaire pour la Navigation à vue d'un robot mobile autonome". M.S. Thesis in Neurosciences ("DEA" in French), Neurosciences, Univ. Aix-Marseille II, 1986.

[16] N. Franceschini, C. Blanes, and L. Oufar, "Passive, non-contact optical velocity sensor". Technical report (in French), FRANCE: ANVAR/DVAR N ${ }^{\circ} 51549$, Paris, 1986

[17] N. Franceschini, A. Riehle, and A. Le Nestour, "Directionally selective motion detection by insects neurons". In Facets of Vision, D.G. Stavenga, R.C. Hardie, Eds. Springer, Berlin, pp. 360-390, 1989.

[18] D. Coombs, and K. Roberts, "Bee-bot: using peripheral optical flow to avoid obstacles". Intelligent Robots and Computer Vision XI, SPIE, vol. 1825, pp. 714-721, 1992.

19] A.P. Duchon, and W.H. Warren, "Robot navigation from a gibsonian viewpoint". In Proceedings of the Int. Conf. on Systems, Man and Cybernetics (San Antonio, Texas, Oct. 2-5), pp. 2272-2277, 1994.

[20] J. Santos-Victor, G. Sandini, F. Curotto, and S. Garibaldi, "Divergent stereo in autonomous navigation: from bees to robots". Int. J. of Computers Vision, no. 14, pp. 159-177 (March), 1995.

[21] K. Weber, S. Venkatesh, and M.V. Srinivasan, "Insect inspired behaviours for the autonomous control of mobile robots". In From Living Eyes to Seeing Machines. Srinivasan and S. Venkatesh, Eds. Oxford University Press, pp. 226-248, 1997.

[22] Argyros, A.A.; D.P. Tsakiris and C. Groyer. 2004. "Biomimetic Centering Behavior for Mobile Robots with Panoramic Sensors". IEEE Robotics and Automation Magazine, special issue on "Panoramic Robotics", Eds. K. Daniilides and N. Papanikolopoulos, vol. 11, pp. 2130, December 2004.

[23] Hrabar S.E., P.I. Corke, G.S. Sukhatme, K. Usher and J.M. Roberts, "Combined optic-flow and stereo-based navigation of urban canyons for a UAV". In Proceedings of the IEEE/RSJ Int. Conference on Intelligent Robots and Systems, (Edmonton, Canada, August 2-6), 2005.

[24] T.R. Neumann, and H.H. Bülthoff, "Insect inspired visual control of translatory flight". In Proceedings of the $6^{\text {th }}$ European Conference on Artificial Life ECAL 2001. Eds. J. Kelemen and P. Sosik, vol. 2159 of LNCS/LNAI, Springer-Verlag, Berlin, pp. 627-636, 2001.

[25] L. Muratet, S. Doncieux, Y. Briere, and J-A. Meyer, "A contribution to vision-based autonomous helicopter flight in urban environments". Robotics and Autonomous Systems, vol. 50, Issue 4, pp. 195-209, 2005.

[26] J. S. Humbert, R. M. Murray, and M. H. Dickinson, "Sensorimotor convergence in visual navigation and flight control systems". In proceedings on the $16^{\text {th }}$ IFAC World Congress ( Prague, Czech Republic, July 4-8), 2005.

[27] Zufferey J-C. and D. Floreano, "Toward 30-gram autonomous indoor aircraft: vision-based obstacle avoidance and altitude control". In proceedings of the IEEE Int. Conference on Robotics and Automation, (Barcelona, Spain, April 18-22), 2005.

[28] F. Ruffier, S. Viollet, S. Amic, and N. Franceschini, "Bio-inspired optical flow circuits for the visual guidance of micro-air vehicles". In Proceedings of the Int. Symposium on Circuits and Systems (Bangkok, Thailand, May 25-28). IEEE, vol. III, pp. 846-849, 2003. 